tion of a large amount of glucose in the urine, the body is losing a substance which has great heat-producing capacities, and which would have taken some part, under normal circumstances, in maintaining the bodily temperature. It has been suggested that the loss of this heat-producing substance may account, wholly or in part, for the subnormal temperature. The condition of the skin, which causes a reduction in the thermolytic processes, may be a part of an effort on the side of the organism to keep the temperature at the normal level. If the subnormal temperature be the result of the excretion of sugar, then some relationship between the amount of this substance present in the urine and the variations in the temperature of the body ought to be found. The temperature should be low when a large amount of sugar is excreted, and nearer the normal when only a small quantity is passed. Investigations have been made with this object, and no relationship between the two has been observed. ${ }^{3}$

In uraemia the bodily temperature is usually slightly below normal, while in uraemic coma it is most commonly normal. There is often, however, in uraemic coma a rise in temperature, which begins a few hours before the death of the patient, and may reach as high as $102^{\circ}$ or $103^{\circ}$. The highest temperature recorded in my series was 103. $8^{\circ}$, which ocourred about twelve hours before the fatal termination of the disease, and which fell to $103^{\circ}$ at the time of death. The rise in temperature may be directly due to the injurious effect on the thermolytic centre of poisonous substances circulating in the blood, or if, as Traube thinks, cerebral oedema and anaemia be the causes of uraemic coma by the action of these two processes upon the regulating centre. Another view is that the rise in temperature may be due to an augmented metabolism incidental to the convulsive movements which occur so often during this form of coma. On the other hand, in many diseases just before the death of the patient there is often a sudden increase in the bodily temperature, which may even rise a degree or two (Fahrenheit) after death has taken place. The cause of this has not been ascertained, and it may be that in uraemic coma the pyrexia is of similar origin.

The temperature charts of patients suffering from intracerebral haemorrhage are very similar to each other, and are very charactêristic of the condition. Coma usually makes its appearance immediately after the onset of the haemorrhage, and with it the temperature suddenly falls below normal. This may be called the temperature of collapse. The next stage is that of reaction, in which the temperature rises either slowly or rapidly, often many degrees above normal. When the first stage is long, the prognosis is very much better than if the temperature rose suddenly and soon after the commencement of unconsciousness. If the patient is going to die in the first stage, a fatal termination occurs almost immediately, or within a very few hours after the onset of the haemorrhage. This stage rarely lasts longer than twelve hours, and is usually only four to six hours in duration. In one of the cases under my observation, however, the temperature remained below $97^{\circ}$ for twenty-four hours, while in another it was below normal for thirty-six hours, and then gradually rose to $107^{\circ}$ in the following three days. At this point the patient died, never having regained consciousness during the whole of the period. The temperature of the stage of collapse is always rather difficult to obtain, as this period is often passed before the patient is seen. A temperature below $97^{\circ}$ seems to be usual, and the lowest which I have found was $95.4^{\circ}$. Bastian ${ }^{4}$ records a rectal temperature as low as $94.4^{\circ}$ in a case in which there was rupture of a large aneurysm in the right corpus striatum with intra-ventricular haemorrhage. As a rule the temperature rises above normal within twelve hours, and the earlier this rise commences the more serious is the prognosis. If the rise in temperature be only a gradual one, that is, reaching its fastigium in about twenty-four hours, and if the temperature be not more than two or three degrees above norma], then the prognosis is more favourable. But if the rise be more sudden and the pyrexia be greater than this then a fatal termination is to be expected.

In cases in which the haemorrhage is very large and also when it occurs in the pons Varolii, the temperature generally rises suddenly and to an extreme height. Although in a pontine haemorrhage there is often hyperpyrexia, a very high temperature is also found associated with large haemorrhages in other parts of the brain. In my series there are two cases of pontine haemorrhage. In one the temperature rose to $105^{\circ}$ in twelve hours, when the patient died ; in the other, a temperature of only $98.4^{\circ}$ was recorded, In the second case, the patient died in the stage of collapse about two hours after the onset of the symptoms. On the other hand, much higher degrees of pyrexia were found in some of my cases in which the haemorrhage occurred in other parts of the brain. In one case of rupture of an aneurysm of the right anterior cerebral artery the temperature rose to $107.8^{\circ}$; in a case of haemorrhage into the corpus striatum to $107^{\circ}$; and in another in which the haemorrhage ruptured into the lateral ventricle to $106.8^{\circ}$. In about a third of the cases of cerebral haemorrhage a temperature of $105^{\circ}$ or above was observed just before the death of the patient. The rapidity of the rise in temperature may sometimes be very great. In one case of haemorrhage into the corpus striatum which extended into the lateral ventricle the temperature was $97.6^{\circ}$ on admission to the hospital, and rose to $106.8^{\circ}$ at the time of death, which occurred within nine hours after the onset of the symptoms.

In comatose patients suffering from fracture of the skull or from concussion the temperature curve is very similar to that found in the last class of cases and therefore requires no special description. In the stage of collapse the temperature was even lower, three cases of $95^{\circ}$ being recorded.

In patients in a comatose condition from alcoholic poisoning, the temperature is nearly always found to be considerably below normal. In one case it was $95^{\circ}$ on admission to the hospital. The patient remained in an unconsciousness condition for about twelve hours, the temperature rising to $97.6^{\circ}$ at the end of that time. According to Dr. H. D. Rolleston, ${ }^{5}$ in some rare instances convulsions may occur and the temperature may rise until death closes the scene, apparently from hyperpyrexia.

The variations in the bodily temperature in cases of opium poisoning, and in patients in the comatose state following an epileptic seizure, I have been unable to ascertain, as my observationson individuals suffering from these two conditions are very few.

From the foregoing considerations it is seen that an observation of the bodily temperature in a comatose state is of considerable value in the prognosis, and is, except under certain circumstances, of comparatively little use in helping to diagnose the cause of the condition when the patient is first seen. In some cases, however, in which the cause of the coma is not evident, an examination of the temperature chart will sometimes clearly indicate its nature, as between uraemic coma and that due to cerebral haemorrhage. If the temperature be above normal soon after the onset of the coma, even only two or three degrees Fahrenheit, then the prognosis is nearly always unfavourable. A very low temperature of $96^{\circ}$ or $95^{\circ}$ at this period indicates usually a fatal termination, as it is generally dependent upon a large cerebral haemorrhage, or some other severe injury to the brain.

REFTRENCES.

1 Saundby, Allbutt's System of Medicine, vol. iii., p. 222. 2 Burdon Sanderson, Allbutt's System of Medicine, vol. i, p. r6o. 3 Sawyer, Clinical Thermometry, 1875, p. 12 ; Foster, BRITISH MEDICAL JouRNAL, 1878, vol. i, p. 78 ; - Rolleston, Allbutt's System of Medicine, vol. ii, p. 848 .

\section{THE STAGE OF INVASION IN MEASLES.}

By R. W. MARSDEN, M.D., M.R.C.P., Physician, Manchester Northern Hospital.

In describing the course of any one of the infectious fevers we are accustomed to divide it into various stages (for example, incubation, invasion, fastigium, and defervescence), and with these terms a definite pathological process is associated in our minds.

According to our present knowledge the infectious disease can only be produced by the entrance into the system of the specific morbific element, and since in many instances the individual attacked has only undergone a very limited exposure, or the infective agent must have been enormously diluted by the conveying medium, we are bound to assume that only a relatively small number of micro-organisms usually effect an entrance. On the other hand, as instanced by those diseases in which the micro-organism has been isolated, we know that as the disease advances a very great multiplication of these organisms takes place within the body, this multiplication being accompanied by the production of various poisonous products on the part of the bacteria, and by the consequent and coincident response from the various cells of the body. It is this response which gives rise 
to the different pathological changes, which are clinically recognized as the signs and symptoms of the disease.

Evidently, then, the length of time which will elapse before a certain sign is manifested, or during which it will remain (even its very occurrence or the severity of the change) must depend on many factors which may be most simply grouped and expressed as the relative "virulence" of the morbific element, and the "vitality" of the cells composing the individual attacked. On this account, then, we might expect that the periods above mentioned would show considerable differences in time of appearance and duration, and from our own experience and that of others we learn that such is the case; for, as T. v. Jürgensen ${ }^{1}$ in his excellent article forcibly remarks, "we know that living beings taking a contagion from other living beings are not governed-at least so far as we can ascertain - by inflexible rules, such as can be applied to the force and frequency of the blows of a steam hammer." Under these circumstances we can hardly refrain from asking ourselves whether there is any advantage in dividing a disease into definite stages, but a little experience soon assures us that the course of the majority of cases is remarkably constant, and the duration, etc., of the various stages of each disease becomes of the greatest assistance in making a differential diagnosis. As the writer I have quoted, however, says, we must "bear in mind that they represent the course of an average case, the accepted length of the stages marking the progressive development of an average infection." Deviations from the standard will often be noted, bat this does not necessarily imply that they are deviations from the " normal," since what we call " normal" only represents the average case.

It is, of course, distinctly unfortunate that in natura phenomena we are so frequently unable to fix a rule which will apply to all circumstances. We learn at first that scarlet fever say commences suddenly, the distinctive exanthem appearing in the subsequent 24 hours, and are then puzzled by occasionally meeting with a case in which its appearance is delayed two, three, or four days. Similarly with measles the teaching becomes engrafted in our minds that the onset occurs gradually, catarrhal symptoms being followed on the fourth day by a distinctive rash, which is first seen about the face. Subsequently we meet with cases in which the prodromata preceding the exanthem are longer or shorter than this period, or the rash makes its appearance in an unusual situation, and many of us immediately begin to question the correctness of our diagnosis. It is with the view of emphasizing these irregularities, as regards the early stages of measles, that I wish to refer to the following cases.

According to v. Jürgensen prodromata may be practically absent in an attack of measles, or constitutional symptoms may arise soon after infection occurs. As an example of the former occurrence I may mention a case reported by Dr. Julius Grober. ${ }^{2}$ The patient was a waiter, 16 years of age, who, with the exception of vaccination and "rheumatism of the joints," had been previously quite well and strong until the day before his admission to the hospital. $\mathrm{He}$ then complained of headache, and the same evening noticed small red spots on the left side of the forehead. Subsequently the rash spread over the nose, cheeks, breast, and back, and the following day was well marked, so that from its appearance; the associated temperature, and the catarrhal symptoms there could be no doubt that the illness was an attack of measles.

From a study of the literature, Dr. Grober states that he has only been able to find one similar case (recorded by Embden) in which the stage of invasion was practically absent. More common, however, are the instances in which the prodromal stage is unusually prolonged, the two following cases serving as examples :

CASE I-A boy, H. B., aged 9, was reported on the night of April 24th to have had a "croupy cough." The next day he seemed quite well, and there was no pyrexia. On April 26th, however, there was a thin serous discharge from the nares, the first alteration in the temperature $\left(99.4^{\circ} \mathrm{F}\right.$.) occurring on the evening of the $27 \mathrm{th}$. On each subsequent day the pyrexia became more marked, whilst on April 28th there was laryngeal cough with slight retraction. On May ist conjunctivitis was added to the symptoms. The rash did not appear till May and, and consisted of small round spots not bigger than the head of a pin, and generally discrete, though larger in some places where several had become confluent. The rash became steadily more intense, the eyelids were oedematous, and the boy suffered from a rather severe attack of measles. On May 4 th the rash was fading, the individual spots being still generally of small size, whilst in many instances small haemorrhages had appeared in them. Bronchitic signs were well marked, and the temperature finally settled on May $\mathbf{x}_{4}$ th.

CASE II.-A youth, G. W., aged . I5, was attacked with measles about the same time as the foregoing. In this case the first indication of on- set was a rise in temperature (100.4 $4^{\circ}$.) on the evening of April 24th. On April ${ }_{25}$ th there was congestion of the throat, with small spots of exudation on the tonsils, and slight rhinitis. For the next eight days the morning temperature was always above $100^{\circ} \mathrm{F}$., whilst the evening temperature was one, two, or more degrees higher. On April 26th there was coryza and conjunctivitis, on' April 27 th herpes of the lips, and on April 29 th the rash was flrst detected, consisting of some faint, blotchy erythemata on the face, and a few scattered macules on the body. The exanthem steadily became more marked though the individual macules remained of small size. On May 2 nd, as in the previous case, haemorrhages were noticed in the spots on the back. The attack was again a severe one, the pyrexia subsiding by lysis, and reaching the normal on May roth.

In both of these cases, then, the exanthem appeared on the sixth day from the onset of pyrexia, whilst in Case I the first indication of illness-that is, "croupy cough"-occurred on the ninth day preceding the eruption. It is thus evident that the stage of invasion in measles may be of very variable duration, and the question arises whether this variability takes place independently of or at the expense of the stage of incubation.

From a careful record of the epidemic in the Faroe Islands Panum $^{3}$ came to the conclusion that the "exanthem appears on the fourteenth day after the reception of the contagium." Like the cases which have been mentioned, he found that some patients suffered from coughs, pains in the eyes, or slight feverishness for six to eight days before the appearance of the exanthem, and that in a few mild cases the prodromal stage either was absent completely or lasted only one to two days. According to this rule, then, though either of the two periods taken separately may vary quite considerably, yet it is always at the expense of each other, their sum being practically a constant number.

Theoretically, one might expect that the absence or diminution of the prodromal stage would, as stated by Panum, occur in the mild cases ; and, conversely, one would expect to meet with early symptoms of disease (that is, a prolonged prodromal stage) in severer cases, the individuals being either predisposed, or the number of micro-organisms introduced at the time of infection being unusually virulent.

Unfortunately I am unable to specify the exact period of exposure in the two cases recorded, yet it seems to me that they tend to support the view I have mentioned, for, though both patients were strong and healthy, one finds that the attack in each case was very similar (thus, prolonged pyrexia, severe general symptoms, small size of individual macules late haemorrhage), and one can hardly help the conclusion that two such unusual attacks from the same source of infection probably owe their "abnormalities" to the virulent properties of the infecting organism.

1 Nothnagel's -Encyclopaedia, Art. Measles, p. $240 ; 2$ Münch. med. Woch., vol. $\dot{i}$, r903, p. $204 ;{ }^{3}$ Virchow's Archiv, r847, Band i, p. 497

\section{SOME REMARKS ON A CASE OF CHLOROMA*.}

\section{By PEVERELL S. HICHENS, M.A., M.D., Oxon. M.R.C.P.,} LoND.

Hon. Physician Northampton General Hospital.

I sHould perhaps offer some apology for spending, I will not say wasting, your time over a case so rare as one of chloroma. In the provinces our lives are severely practical, and we cannot as a rule afford ourselves the luxury of investigating the by-ways and hidden places of medicine.

A practitioner may be pardoned for knowing little or nothing about a case of an occurence so infrequent that he is almost sure never to meet one in the course of his lifetime.

But there are cases of such rarity, or supposed to be of such rarity, that they ought not to go unrecorded, and chloroma is one of them. Since the disease was first described by Allan Burns in $1823,{ }^{5} \mathrm{I}$ believe only 28 cases have been published, and hardly any of these have been diagnosed during life. The case that I am about to describe to you was unfortunately no exception to this rule. Though the patient did not live long enough to develop some of the most characteristic symptoms of this obscure disorder, yet I think I might have succeeded in arriving at a correct diagnosis if $I$ had known more of the literature concerning former cases.

I have ventured, therefore, to bring this case before you today with some general remarks on the disease of which it was

* Read before the South Midland Branch of the British Medical 